\title{
Diseño, desarrollo e implementación de una celda de manufactura didáctica para ingeniería mecatrónica en la UPTx
}

\section{Design, development and implementation of a didactic manufacturing cell for mechatronic engineering at UPTx}

\author{
CANO-CORONA, Ariana $\dagger^{*}$, PEREZ-SERRANO, Froylan, AGUILAR-AGUILAR, Álvaro y \\ HERNANDEZ-ZEMPOALTECATL, Rodrigo
}

Universidad Politécnica de Tlaxcala, Programa Académico de Ingeniería Mecatrónica

ID $1^{\mathrm{er}}$ Autor: Ariana, Cano-Corona / ORC ID 000000033456 2303, CVU: X_acano100364

ID $1^{\text {er }}$ Coautor: Froylan, Perez-Serrano / CVU: 377744

ID $2^{\text {do }}$ Coautor: Álvaro, Aguilar-Aguilar / ORC ID 0000-0001-8050-8055

ID $3^{\text {er }}$ Coautor: Rodrigo, Hernandez-Zempoaltecatl / ORC ID 0000-0002-0677-474X

DOI: $10.35429 / J T D .2019 .11 .3 .9 .13$

Recibido: 24 de Julio, 2018; Aceptado 11 de Septiembre, 2018

\begin{abstract}
Resumen
En el presente trabajo se muestran los fundamentos, las principales actividades desarrolladas y los resultados hasta ahora obtenidos por docentes del programa académico de ingeniería mecatrónica con el proyecto de diseño, e implementación de una celda de manufactura didáctica para el programa académico de ingeniería mecatrónica en el laboratorio de máquinas-herramientas-CNC del lt2 en la UPTx. Dicha celda es una muestra del reforzamiento y potencialización que pueden llevar a cabo las instituciones de educación superior para generar transferencia tecnológica con el sector empresarial de la región. La integración de la celda de manufactura didáctica se llevó a cabo como un proyecto académico en el que se aprovecharon los equipos ya existentes en los diferentes laboratorios del programa educativo de ingeniería mecatrónica y se integró con el diseño y elaboración de los faltantes para el desarrollo completo. Los principales beneficios que se obtuvieron son el fortalecimiento de las capacidades y competencias que los estudiantes pueden lograr durante el periodo de su formación profesional en la Universidad, así como familiarizar al estudiante con los ambientes industriales, en una segunda etapa se pretende utilizar dicha celda como recurso de educación continua con las empresas de la región, así como con los alumnos egresados.
\end{abstract}

Celda de manufactura, Didáctica, Mecatrónica

\begin{abstract}
The present work presents the fundamentals, the main activities developed and the results so far obtained by teachers of the academic program of mechatronic engineering with the design project, and implementation of a didactic manufacturing cell for the academic program of mechatronic engineering in the laboratory of machine tools-CNC of the lt 2 in the UPTx. This cell is a sample of the reinforcement and potentialization that higher education institutions can carry out to potentiate the technological transfer with the business sector of the region. The integration of the didactic manufacturing cell was carried out as an academic project in which the existing equipment in the different laboratories of the educational mechatronic engineering program was used and integrated with the design and elaboration of the missing ones for the complete development. The main benefits obtained are the strengthening of the skills and competences that students can achieve during the period of their professional training at the University, as well as familiarizing the student with industrial environments, in a second stage it is intended to use said cell as Continuous education resource with the companies of the region, as well as with the graduated students
\end{abstract}

Manufacturing cell, Teaching, Mechatronics

Citación: CANO-CORONA, Ariana, PEREZ-SERRANO, Froylan, AGUILAR-AGUILAR, Álvaro y HERNANDEZZEMPOALTECATL, Rodrigo. Diseño, desarrollo e implementación de una celda de manufactura didáctica para ingeniería mecatrónica en la UPTx. Revista del Desarrollo Tecnológico. 2019 3-11: 9-13

\footnotetext{
* Correspondencia del Autor (Correo electrónico: ariana.cano@uptlax.edu.mx)

$\uparrow$ Investigador contribuyendo como primer autor. 


\section{Introducción}

En este documento se presenta el diseño e implementación una celda de manufactura didáctica en el laboratorio de MáquinasHerramientas-CNC de la Universidad Politécnica de Tlaxcala, con la principal intención de apoyar en el desarrollo de capacidades y competencias de los estudiantes de Ingeniería Mecatrónica, este diseño se realizó utilizando los equipos existentes de los laboratorios de la carrera de ingeniería mecatrónica. La importancia del desarrollo e implementación de este proyecto recae en el fortalecimiento de las competencias profesionales a nivel ingeniería que deberán poner en práctica los estudiantes y egresados de este Programa Académico.

Académicamente, el valor agregado consiste en fortalecer las líneas de investigación y la gestión de proyectos por parte de los profesores que integran los cuerpos académicos de la carrera $\mathrm{y}$, por otra parte, motivar a los estudiantes en la participación de proyectos que promuevan la aplicación de conocimientos en beneficio del sector productivo y social del estado de Tlaxcala y la región.

La competitividad a nivel regional $\mathrm{y}$ nacional ejerce presión a las industrias del sector manufacturero, por lo tanto, es necesario e indispensable proporcionar una educación integral a los futuros ingenieros para que manejen los recursos tecnológicos y humanos que les permitan competir dentro de estos mercados y fortalecer su profesionalización.

Recientemente las empresas han centrado sus esfuerzos en el aumento de la productividad por medio del uso y aprovechamiento de las nuevas tecnologías; por lo que la integración de todas las áreas de la empresa es su opción viable, estratégicamente hablando para incrementar su productividad, de esta forma al incentivar a los estudiantes a la integración de los sistemas se replica la intención de aportación hacia la mejora de las empresas.

Los principales aspectos que se reforzaran con el desarrollo del proyecto, dadas las necesidades y oportunidades que las industrias de la región requieren, consisten en:
- Ofertar cursos de capacitación continua a las empresas, así como a alumnos egresados.

- $\quad$ Reforzar y potencializar la transferencia de tecnología que la institución aporta a la región.

- Aprovechar los equipos existentes en los diferentes laboratorios del programa educativo de ingeniería Mecatrónica y hacer una integración de los mismos para familiarizar al estudiante con un ambiente industrial.

- $\quad$ Fortalecer las líneas de investigación y la gestión de proyectos por parte de los profesores que integran los cuerpos académicos de la carrera.

- Motivar a los estudiantes en la participación de proyectos que promuevan la aplicación de conocimientos en beneficio del sector productivo y social, así como la transferencia de tecnología.

\section{Diseño de la celda de manufactura}

Con la intención principal de fortalecer las competencias profesionales de los estudiantes de ingeniería mecatrónica, se diseñó una celda de manufactura didáctica que permitiera emular procesos de manufactura industrial.

Inicialmente se llevó a cabo una investigación técnica de los elementos mecánicos, eléctricos y electrónicos que integrarían la celda y que fueran los más adecuados para permitir un escalamiento del sistema (ver Tabla 1).

\begin{tabular}{|l|l|l|}
\hline \multicolumn{2}{|c|}{ Elementos } & \multicolumn{1}{c|}{ Características } \\
\hline 1 & Conveyor & Tablilla \\
\hline 2 & Motor & $1 / 4 \mathrm{Hp}, 220-440$ Vac, 3fase \\
\hline 3 & Cuerpo de lámina & Calibre 16 \\
\hline 4 & Maquinados & Flechas, placas, etc. \\
\hline 5 & Tablero eléctrico & \\
\hline 6 & Variadorde frecuencia & \\
\hline 7 & Sensores de inicio y fin & \\
\hline 8 & $\begin{array}{l}\text { Controlador Lógico } \\
\text { Programable }\end{array}$ & PLC S7-1200 \\
\hline
\end{tabular}

Tabla 1 Características de los elementos que integrarán la celda de manufactura didáctica

Fuente: Elaboración Propia

De acuerdo con el análisis se acordó que debido a las dimensiones donde sería instalada la celda de manufactura, el transportador modular (bandas) debería ser de $100 \mathrm{~mm}$ de ancho por 2000mm de largo y $900 \mathrm{~mm}$ de alto.

CANO-CORONA, Ariana, PEREZ-SERRANO, Froylan, AGUILARAGUILAR, Álvaro y HERNANDEZ-ZEMPOALTECATL, Rodrigo. Diseño, desarrollo e implementación de una celda de manufactura didáctica para ingeniería mecatrónica en la UPTx. Revista del Desarrollo Tecnológico. 2019 
El material utilizado para el transportador fue de lámina negra calibre \#14, el conveyor fue de bisagra simple de cadenas rectas para grandes cargas, con pasadores de acero inoxidable endurecido al vacío.

Este tipo de conveyor presenta ventajas de alta resistencia, así como estructura estándar con malla transportadora y el ancho de bisagra está disponible para varios tipos de cadena.

De igual forma las tablillas presentan bajo nivel de ruido y coeficiente de fricción, no se absorben líquidos y tienen buena resistencia química y al desgaste, finalmente al ser de estructura cerrada son más fáciles de limpiar y desinfectar.

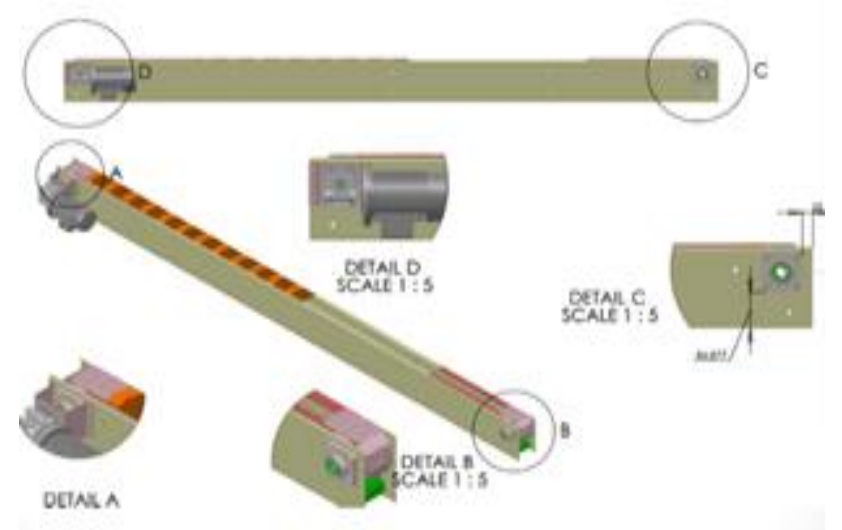

Figura 1 Diseño en 3D del transportador modular Fuente: Elaboración Propia

\section{Desarrollo e implementación de la celda de manufactura}

Como se observa en la figura 2, con apoyo del software Solid Works 2019, se propuso una configuración en "L" para poder realizar la carga y descarga del material a maquinar en el torno CNC ya que en una segunda etapa se integrará un brazo de robot Motoman.

El movimiento de los bandas es en ambos sentidos, a través de un engrane de transmisión $\mathrm{y}$ utilizando un sistema de retorno seguro y eficaz, ya que el posicionamiento depende del modelo mecánico.

La finalidad de flexibilizar la celda de manufactura se enfocó en configurar procesos diferentes y poder diversificar el aprendizaje de los estudiantes en el desarrollo de las prácticas.

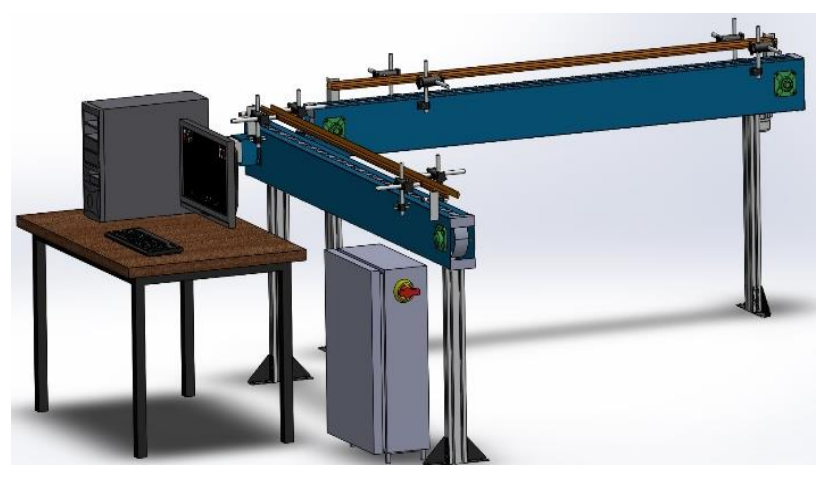

Figura 2 Diseño de la celda en 3D con SolidWorks Fuente: Elaboración Propia

El motor eléctrico trifásico cuya potencia es de $0.25 \mathrm{~W}$ y gira a 1370 revoluciones por minuto (RPM) con conexión delta a $220 \mathrm{VCA}$, está acoplado a una caja reductora de velocidad que tiene como proporción 20:1, velocidad que es regulada mediante un variador de frecuencia, dispositivo electrónico programable trifásico que permite controlar la velocidad del motor mediante la variación del valor de la frecuencia.

Las variables más importantes que se consideraron para el funcionamiento adecuado del variador fueron, los pulsos de entrada $y$ salidas digitales, la sobrecarga termoeléctrica, la frecuencia portadora, la limitación de la corriente, la sobretensión y el par motor.

El control general del sistema se realizó con un controlador SIMATIC S7-1200 controlador lógico programable PLC con CPU $1214 \mathrm{C}$ el cual cuenta con 14 entradas digitales tipo sumidero/fuente a tensión nominal de 24 VDC a $4 \mathrm{~mA}$ y 10 salidas digitales tipo relé, rango de voltaje de 5 a 30 VDC o de 5 a 250 VAC con una corriente máxima de 2 Amp. y 2 entradas analógicas tipo tensión (unipolares) con rango de tensión de 0 a $10 \mathrm{~V}$ y resolución de 10 bits.

La resolución del tamaño de imagen de proceso del CPU es de 1024 para entradas y las salidas, con memoria de trabajo de $50 \mathrm{~KB}$.

Finalmente los 4 sensores implementados en el sistema son de tipo fotoeléctricos de la serie C2DP-11P con alcance de censado es de $110 \mathrm{~mm}, \mathrm{PNP}$

Las características anteriores permitieron la configuración de la velocidad y control adecuados, tanto de avance como del retroceso de las bandas. 


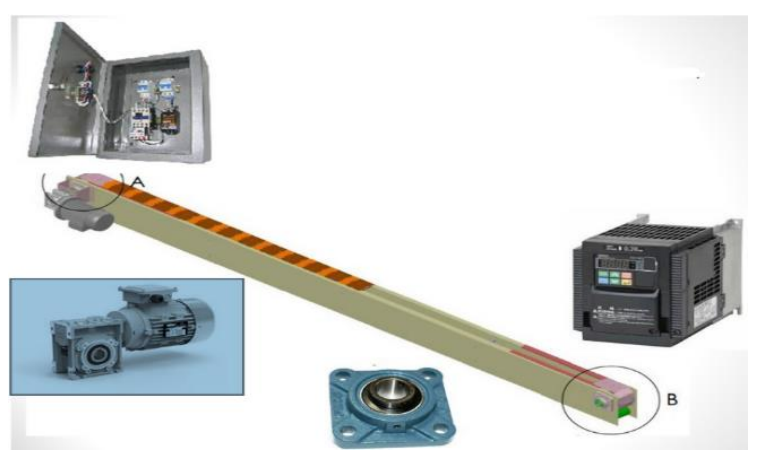

Figura 3 Elementos que integrarán la celda de manufactura

Fuente: Elaboración Propia

Los módulos transportadores llevan unas guías laterales para apoyar el recorrido óptimo de las piezas sobre la banda y facilitar su detección por medio de los sensores

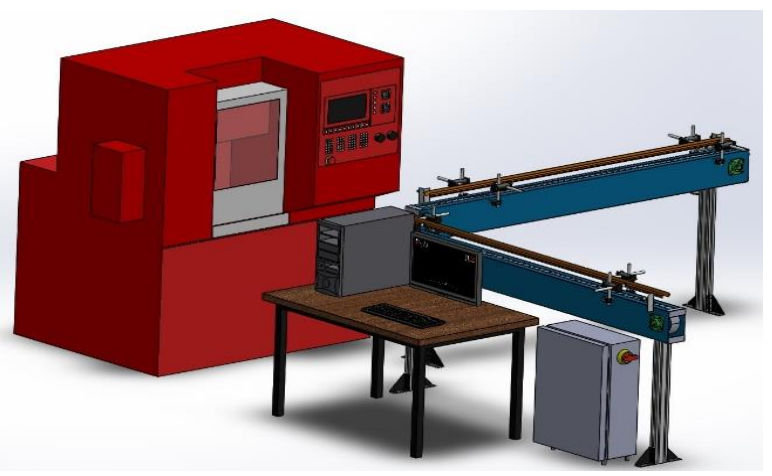

Figura 4 Diseño de integración de la celda de manufactura Fuente: Elaboración Propia

\section{Resultados}

Con el desarrollo e implementación alcanzado de la integración de la celda de manufactura, se ha impactado directamente el desarrollo y formación de competencias de los estudiantes del Programa Educativo de Ingeniería Mecatrónica, ya que actualmente los alumnos pueden hacer uso de estos equipos en los diferentes momentos de su formación profesional, y por lo tanto pueden interactuar y trabajar con maquinaria y equipos aptos para la automatización industrial (ver figura 5).

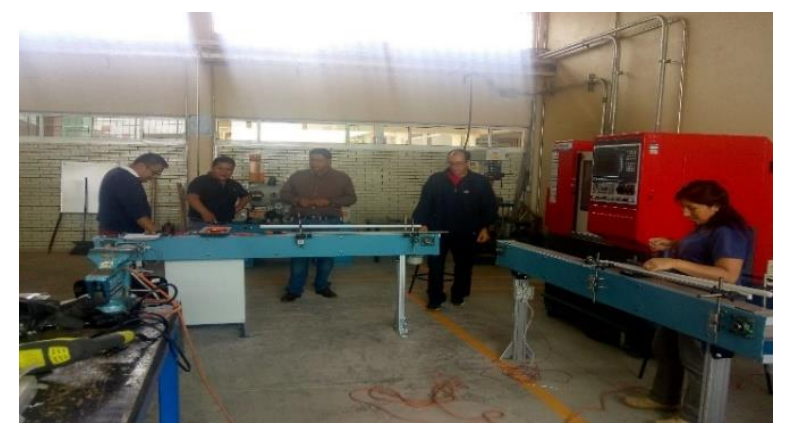

Figura 5 Implementación física de la celda de manufactura, Elaboración Propia
Como se observa en la tabla 2, se enlistan las asignaturas del plan vigente del programa educativo que pueden hacer uso de la celda de manufactura.

\begin{tabular}{|l|l|}
\multicolumn{1}{|c|}{ Asignatura } & $\begin{array}{c}\text { Cuatrimestre } \\
\text { en que se } \\
\text { cursa }\end{array}$ \\
\hline Programación de periféricos & sexto \\
\hline Sensores y acondicionamiento de señales & cuarto \\
\hline Automatización industrial & sexto \\
\hline Adquisición y procesamiento de señales & octavo \\
\hline Redes industriales & noveno \\
\hline Procesos de manufactura & segundo \\
\hline Sistemas CAE & séptimo \\
\hline Sistemas CAM - CNC & octavo \\
\hline Sistemas electrónicos de interfaz & cuarto \\
\hline Controladores Lógicos Programables & quinto \\
\hline Programación estructurada & quinto \\
\hline Programación de sistemas embebidos & octavo \\
\hline Sistemas Avanzados de manufactura & noveno \\
\hline
\end{tabular}

Tabla 2 Asignaturas del mapa curricular en las que se daría utilidad a la celda de manufactura didáctica, Elaboración Propia

En una segunda etapa del proyecto se desarrollarán manuales específicos de prácticas de laboratorio de dichas asignaturas y que son indispensables para uso de los docentes y estudiantes que lo requieran para poder manipular los equipos que hasta ahora han sido integrados.

Es importante recalcar que también se tiene programado el uso de la celda de manufactura para ofertar cursos de capacitación hacia el sector industrial de la región, así como el desarrollo de prácticas con egresados y que sirvan para las diversas asignaturas que conforman el plan de estudios de esta ingeniería.

Actualmente con la integración del 90\% de los equipos se está cumpliendo con el fortalecimiento de las capacidades y competencias que los estudiantes pueden lograr durante el periodo de su formación profesional en la Universidad Politécnica de Tlaxcala.

La integración del robot motoman está pendiente, por trámites administrativos del traslado hacia el laboratorio de máquinasherramientas. Finalmente se ha generado una alta expectativa de participación de los estudiantes en el desarrollo de proyectos que promueven la aplicación de conocimientos en beneficio del sector productivo y social del sector productivo de la región.

CANO-CORONA, Ariana, PEREZ-SERRANO, Froylan, AGUILARAGUILAR, Álvaro y HERNANDEZ-ZEMPOALTECATL, Rodrigo. Diseño, desarrollo e implementación de una celda de manufactura didáctica para ingeniería mecatrónica en la UPTx. Revista del Desarrollo Tecnológico. 2019 


\section{Agradecimiento}

Agradecemos el apoyo otorgado por el Programa de Desarrollo Profesional PRODEP para el nivel superior. A la Universidad Politécnica de Tlaxcala, por las facilidades otorgadas para la realización del trabajo.

\section{Conclusiones}

El diseño, desarrollo e implementación de este proyecto en el laboratorio de máquinasherramientas de la carrera de Ingeniería Mecatrónica en la Universidad Politécnica de Tlaxcala ha sido de gran importancia ya que ha permitido que los docentes pongamos en práctica el trabajo colaborativo y seamos ente generador de desarrollo tecnológico al interior de la universidad, con la finalidad de fortalecer las competencias de los estudiantes en los diferentes niveles de su formación profesional.

Se está avanzando en el desarrollo de prácticas en los laboratorios, que sin duda son una parte muy importante en el aprendizaje de los estudiantes ya que les permite comprobar lo que dicta la teoría, además de acercarlos a una realidad laboral en la que en un futuro se habrán de desempeñar de manera profesional. También se observa que el impacto académico de esta celda de manufactura es alto ya que son 13 asignaturas del mapa curricular vigente de ingeniería mecatrónica en las que se desarrollan prácticas cumpliéndose así con los propósitos de desarrollo e implementación de este proyecto.

\section{Referencias}

Aguirre, A. \& Gomez Suarez J., Ramirez Bernal, F. Coronel Bustamante, C. (2013). Celda de manufactura flexible. 10.13140/2.1.4818.9449.

Alvarez Rodriguez, D.A., Chaquea León M.P. "Proceso de reingeniería de la celda flexible de manufactura didáctica del laboratorio de automatización de la fundación Universidad de América". Tesis de Grado, Bogotá, Colombia (2018).

Cano Corona A., Hernández Zempoaltecatl R., Pérez Serrano F., Aguilar Aguilar A. "Integración de celda de manufactura didáctica en Ingeniería Mecatrónica" Congreso Nacional de Mecatrónica, Guanajuato, México 11 de Octubre de 2013.
Córdoba Nieto E. "Manufactura y Automatización". Ingeniería e Investigación, vol. 26. $\mathrm{N}^{\circ}$ 3, Diciembre 2006 página 120-128.

Ficha técnica de conveyor, 08/07/2019, recuperado de: https://www.systemplastsmart guide.com/INT/Spanish-Smart-Guide/11100A/\#

Ficha técnica de interruptores automáticos magnetotérmicos, 08/07/2019, recuperado de: https://new.abb.com/low-voltage/es/ productos/aparamenta-modular/interruptoresautomaticos-magnetotermicos/interruptoresautomaticos-magnetotermicos-para-usoterciario-sh-200

Ficha técnica de sensores, 08/07/2019, recuperado de: http://www.webddigital.com/fabricantes/optex/ pdf/sensores/c_c2.pdf

García Gallardo J.I., Cano Corona A., Hernández Zempoaltecatl R. "Transferencia de tecnología para integración de celda de manufactura didáctica" Congreso Internacional de Investigación de AcademiaJournals.com, Celaya, México 08 de Noviembre de 2013.

López G., "Fundamentos de control inteligente de la manufactura flexible". Instituto Politécnico Nacional, 2008.

Manual de Controlador Lógico Programable PLC S7-1200, 08/07/2019, recuperado de: https://w5.siemens.com/spain/web/es/industry/a utomatizacion/sce_educacion/DocumentacionDidactica/Documents/SIMATIC\%20S71200R.p df

Manual de motor eléctrico, 08/07/2019, recuperado de: https://new.abb.com/products/1SAM250000R1 010/ms 116-10-manual-motor-starter

Mejía Moncayo C., "Modelo para definición del layout de una celda de manufactura a través de optimización”. Bogotá, Colombia (2012).

Puga Velázquez E. S. Foyo Valdés S. A., Peñaloza Mejía O., "Modelado de una celda de manufactura integrada por computadora”. Conciencia tecnológica, Un. 39 Enero-Junio 2010, pp. 23-30. ITA, México. 